\title{
Methode zur Bestimmung der Emission von Diphenylmethan-4,4'-diisocyanat (MDI) aus isocyanatgebundenen Holzwerkstoffen
}

\author{
P. Mattrel, K. Richter
}

Es wird ein Analysenverfahren vorgestellt mit dem Diphenylmethan-4,4'-diisocyanat (MDI)- und 4,4'-Diamino-diphenylmethan (MDA)-Emissionen aus Isocyanatklebstoffen gemeinsam als MDA erfaßt und mittels HPLC quantitativ bestimmt werden können. Die Methode erlaubt gegenüber bisherigen, meist photometrischen Bestimmungen eine vollständigere und verfeinerte Ansprache möglicher Isocyanatemissionen. Die praktische Anwendung der Analytik auf in Prüfzellen exponierte isocyanathaltige Produkte (Spanplatten, frische Klebstofffläche) unter "worst-case"-Bedingungen ergab keine bzw. eine nur im Bereich der Nachweisgrenze liegende, kurzzeitige MDI/MDA-Emission. Damit werden bisherige wissenschaftliche Ergebnisse untermauert, die Isocyanatemissionen sowohl für die Verarbeitung als auch für die Verwendung von polyurethanverklebten Holzprodukten und Holzwerkstoffen ausschliessen.

\section{Method for the determination of 4,4'-dipheny/methane diisocyanate} (MDI)-emissions from diisocyanate-bonded wood products

A method for the determination of 4,4'-diphenylmethane diisocyanate (MDI) and 4,4'-methylenedianiline (MDA) in air is presented. The air sample is drawn through an impinger containing diluted hydrochloric acid as absorption solution. MDI hydrolyzes quantitatively to MDA and is determined together with MDA absorbed from the air by high performance liquid chromatography after derivatization with 2,4-dinitro-1-fluorobenzene. MDA was included in the analysis because of its carcenogenic potency and to avoid an eventual loss of MDI due to hydrolysis during exposure and sampling. The method is sensitive (detection limit ca. $0.5 \mu \mathrm{g} \mathrm{MDI} / \mathrm{MDA} / \mathrm{m}^{3}$ ) and specific; other diisocyanates as tolylene-2,4-diisocyanate (2,4-TDI), tolylene-2,6-diisocyanate (2,6-TDI), 1,6-diisocyanatohexane (HDI) and isophorone diisocyanate (IPDI), respectively the corresponding diamines, do not interfere. MDI-bonded particleboard and a polymeric MDI adhesive were exposed in a test chamber under worst case-conditions (particleboard: high surface/air volume ratio $\left(2 \mathrm{~m}^{2} / \mathrm{m}^{3}\right)$ without air exchange; adhesive: large open surface in a small test chamber $\left(0.16 \mathrm{~m}^{2} / 0.01 \mathrm{~m}^{3}\right)$, thick adhesive layer $\left(600 \mathrm{~g} / \mathrm{m}^{2}\right)$ and sampling start immediately after spreading). No MDI/MDA-emission was found in the case of particleboard. During the first two hours of exposure the adhesive emitted a very low amount of MDI/MDA near the detection limit.

\section{1}

\section{Einleitung}

Die Beurteilung der Eignung von Klebstoffen für Holzverbindungen erfolgt heute nicht mehr allein nach physikalischtechnischen sowie ökonomischen Gesichtspunkten, sondern

\section{P. Mattrel, K. Richter}

Eidgenössishhe Materialprüfungs- und Forschungsanstalt (EMPA) Ueberlandstraße 129 CH-8600 Dübendorf (Schweiz) stützt sich vermehrt auch auf Angaben zu klebstoffspezifischen Umweltauswirkungen ab. Von primärem Interesse sind hier im Sinne des Arbeitsschutzes Gesundheitsbeeinträchtigungen, die während der Herstellung oder Applikation eines Klebers auf Arbeiter und Ausführende auftreten können. Während der Gebrauchsphase können gasförmige Emissionen aus der Klebstoffuge möglicherweise über längere Zeiträume in die Umgebungsluft abgegeben werden. Weiter bestimmen Hilfsund Zusatzstoffe ganz wesentlich die Möglichkeiten der stofflichen oder thermischen Verwertung von Holzprodukten am Ende der ersten Nutzungsphase eines Produktes.

Vor diesem Hintergrund müssen auch die Isocyanat- und Polyurethan (PUR)-Klebstoffe beurteilt werden, die heute in der Holzwerkstoffindustrie insbesondere dort zum Einsatz kommen, wo konventionelle Klebstoffsysteme nicht alle Anforderungen erfüllen.

Ausgehend von einzelnen Spezialanwendungen im Flugzeugbau wurde in den siebziger Jahren der industrielle Einsatz von Isocyanaten auf der Basis von "polymerem" MDI (PMDI) zur Herstellung von Spanplatten vollzogen (Ernst 1985). Heute nehmen die Isocyanate in reiner Form oder in Mischung mit anderen Komponenten mit rund 15\% einen konstanten Marktanteil neben den Polykondensationsharzen ein. Im konstruktiven Holzbau dagegen haben Polyurethan-Klebstoffe bisher vergleichsweise wenig Beachtung gefunden, obwohl ihnen positive Entwicklungschancen zugesprochen werden (Hinterwaldner, Kreibich 1987). Einzelne Aussenanwendungen von PUR-Systemen in der Schweiz haben sich sehr gut bewährt. Vor kurzem wurden an der FMPA Stuttgart umfangreiche Zulassungsuntersuchungen mit einem Einkomponenten PUR-Klebstoff erfolgreich abgeschlossen (Radovic, Goth 1994), so dass derartige Polyadditionsklebstoffe auch im bundesdeutschen Holzbau eine verstärkte Bedeutung erlangen dürften.

Neben den hervorragenden klebetechnischen Eigenschaften (großes Reaktionsvermögen, flexible Einsatzmöglichkeiten für Holz-Holz- und Komposit-Verklebungen) und günstigen Feuchteverhalten (Abbindung bei hoher Holzfeuchte, gute Feuchtebeständigkeit, gewisse Hydrophobierung) dürfte bei der Spanplattenverleimung auch der Verzicht auf Formaldehyd zum Durchbruch der Isocyanate beigetragen haben. Weitere positive Umweltaspekte lassen sich aus der Tatsache ableiten, dass bei der Massivholzverleimung die Anforderungen an die Lamellenfeuchte weniger hoch sind und damit Trocknungsenergie eingespart werden könnte. Bei der Spanplattenproduktion allerdings lässt sich dieser theoretische Vorteil aus produktionstechnischen Gründen derzeit noch nicht ausnutzen, die Späne werden auch für die MDI-Verleimung auf $1 \%$ Holzfeuchte abgetrocknet. Insgesamt geringere

Verbrauchsmengen, kürzere Aushärte- und Preßzeiten und die guten Kombinationsmöglichkeiten der Isocyanate mit natürlichen Extrakten, z.B. auf der Basis von Rindentanninen (Frisch et al. 1983) sind weitere Punkte, die bei einer ganzheitlichen Umweltbewertung der Isocyanate einbezogen werden müssen. 
Als ökologisch kritisch dagegen sind die reaktiven, aromatischen Komponenten der Klebstoffe selbst und ihre möglichen Wechselwirkungen mit der Umwelt anzusehen. Isocyanate sind stark toxische Verbindungen, die als gefährliche Arbeitsstoffe eingestuft und mit einem entsprechend tiefen MAK-Wert von $0,05 \mathrm{mg} / \mathrm{m} 3$ (= 0,005 vol ppm) (DFG 1992) für MDI belegt sind. Sie können zu Überempfindlichkeitsreaktionen allergischer Art, dem sogenannten Bronchialasthma führen, das sich in Reizerscheinungen der Schleimhäute, Hustenreiz und, in extremen Fällen, Atemnot äußert. In der Schweiz sind im industriellen Bereich Isocyanate die häufigste Ursache von Berufsasthma (Jost et al. 1990).

Die Spanplattenherstellung unterliegt nach dem Bundesimmissionsschutzgesetz der Genehmigungspflicht, so daß Messungen der Arbeitsplatzemissionen von amtlich zugelassenen Stellen erfolgen. Überschreitungen der tiefen Grenzwerte bei der PMDI-Verarbeitung sind bisher nicht bekannt geworden.

Unterschiedlich beurteilt wird die Frage, ob aus den verpressten Platten bzw. Klebstoffugen infolge nicht umgesetzter Monomere MDI oder Umwandlungsprodukte an die Umgebungsluft abgegeben und damit zu einer Gesundheitsgefährdung für die Verbraucher werden können. Aufgrund des geringen Dampfdrucks von MDI (die Sättigungskonzentration bei Raumtemperatur entspricht in etwa dem MAK-Wert) erscheint eine Emission aus Spanplatten wenig wahrscheinlich. Bei der Simulation einer Wohnraumsituation mit mindestens drei Monate alten PMDI-gebundenen Spanplatten konnte bei einer Nachweisgrenze von $0,1 \mu \mathrm{g} / \mathrm{m}^{3}$ keine MDI-Emission festgestellt werden (Kehl 1990). Messungen an frisch gepreßten Spanplatten in einer 20 Liter Prüfkammer mit einer Belegung von $5 \mathrm{~m}^{2} / \mathrm{m}^{3}$ ergaben eine MDI Konzentration von $0,01 \mu \mathrm{g} / \mathrm{m}^{3}$ (Schmidtke, Seifert 1990), während bei zwei Stunden alten Platten auch bei erhöhten Temperaturen $\left(60^{\circ} \mathrm{C}\right)$ keine MDIAbgabe festgestellt wurde (Marutzky, Menzel 1985). Von baubiologischer Seite jedoch werden immer wieder Vermutungen geäußert, daß Emissionen aus ungebundenen Isocyanaten aus Platten abgegeben und eine Gesundheitsgefahr für die Raumbenutzer darstellen (z.B. Schwarz 1991).

Aufrund dieser sowohl für die Verarbeiter als auch für Verbraucher unbefriedigenden Sachlage wurden auch an der EMPA in den letzten Jahren Emissionsmessungen an isocyanatgebundenen Holzwerkstoffen durchgeführt. Wegen des nachfolgend beschriebenen Chemismus der Isocyanate erschien es nötig, neue und verfeinerte Bestimmungsmethoden zu entwickeln, um die Aussagefähigkeit der Analysen weiter zu verbessern. Die verbesserte Methodik und die damit erhaltenen Ergebnisse sind als Beitrag zu einer weiteren Versachlichung der Diskussion um MDI-Emissionen nachfolgend beschrieben.

\section{2}

\section{Chemie der Isocyanate}

Isocyanate sind äußerst reaktionsfähige Verbindungen. Für die Herstellung und Verarbeitung von Polyurethan sind vor allem folgende Reaktionen von Bedeutung:
1) $\mathrm{R}-\mathrm{N}=\mathrm{C}=\mathrm{O}$
$\mathrm{R}^{\prime}-\mathrm{OH}$
Isocyanat
Alkohol
$\rightarrow \mathrm{R}-\mathrm{NH}-\mathrm{CO}-\mathrm{O}-\mathrm{R}^{\prime}$ Carbamat (Urethan)
2) $\mathrm{R}-\mathrm{N}=\mathrm{C}=\mathrm{O}+\underset{\text { Wasser }}{\mathrm{H}_{2} \mathrm{O}} \rightarrow \underset{\text { Amin }}{\mathrm{R}-\mathrm{NH}_{2}+\mathrm{CO}_{2}}$
3)
$\mathrm{R}-\mathrm{N}=\mathrm{C}=\mathrm{O}$
$+\mathrm{R}^{\prime}-\mathrm{NH}_{2} \rightarrow$
Amin
$\mathrm{R}-\mathrm{NH}-\mathrm{CO}-\mathrm{N}$
Harnstoff-Derivat

Polyurethane werden beim Zweikomponenten-Verfahren durch Umsetzung von Di- oder Polyisocyanaten mit

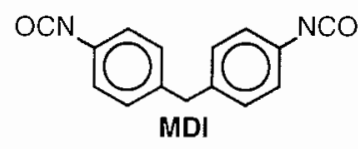<smiles>Nc1ccc(-c2ccc(N)cc2)cc1</smiles><smiles>Cc1ccc([N+](=O)[O-])cc1[N+](=O)[O-]</smiles>

2,4-TDI

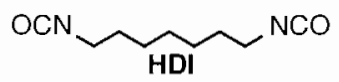<smiles>CC1(C)CC([N+](=O)[O-])CC(C)(CN=O)C1</smiles>

Bild 1. In die Untersuchung einbezogene Diisocyanate und Diamine Fig. 1. Diisocyanates and diamines included in method development

\section{Polyalkoholen gemäss Reaktion 1 hergestellt. Beim} Einkomponenten-Verfahren dagegen härten Vorpolymerisate, die freie Isocyanat-Gruppen enthalten, mit Umgebungswasser aus. Dabei bilden sich aus einem Teil der Isocyanate Amine (Reaktion 2), die ihrerseits wieder mit Isocyanat-Gruppen unter Bildung von Harnstoff-Derivaten reagieren (Reaktion 3). Die gebräuchlichsten Diisocyanate sind in Bild 1 dargestellt.

Für die Bindemittel auf Isocyanatbasis wird in der Holzindustrie als Isocyanatkomponente fast ausschließlich PMDI eingesetzt (Schriever 1986). Es handelt sich dabei um ein Gemisch von monomerem MDI (30-80 Massen-\%) mit seinen Oligomeren und Homologen. Die Isocyanatgruppen des Bindemittels reagieren einerseits mit dem Umgebungswasser zu Polyharnstoffen und andererseits mit den Hydroxylgruppen des Holzes (Lignin, Cellulose) unter Ausbildung von Urethangruppen. Etwa die Hälfte des PMDI setzt sich zu Polyharnstoff um (Frisch et al. 1983).

Aufgrund der Reaktivität der Isocyanate besteht die Möglichkeit, daß sich zumindest ein Teil des MDI mit Umgebungswasser umsetzt und sich dadurch einer Isocyanatspezifischen Bestimmung entzieht. Da das dabei entstehende 4,4'-Diamino-diphenylmethan (MDA) als kanzerogen eingestuft wird (DFG 1992), wäre eine Emission von MDA oder eine Hydrolyse von emittiertem MDI zu MDA nicht nur von analytischer, sondern auch von gesundheitlicher Bedeutung. Der Frage wird deshalb auch bei der Begründung des MAK-Werts für MDI nachgegangen. Bei Konzentrationen von bis zu $5 \mathrm{mg}$ $\mathrm{PMDI} / \mathrm{m}^{3}$ konnte kein MDA nachgewiesen werden bei einer Nachweisgrenze von $0,005 \mathrm{mg} \mathrm{MDA} / \mathrm{m}^{3}$ (DFG 1992). Dies wird mit der Ausbildung einer Schutzschicht aus Polyharnstoff erklärt, die sich an der Oberfläche des bei diesen hohen Konzentrationen als Aerosol vorliegenden MDI bildet. Bei Emissionsmessungen liegen jedoch andere Verhältnisse vor, da MDI gasförmig und nicht als Aerosol auftritt und bei der geringen Sättigungskonzentration $\left(0,05 \mathrm{mg} \mathrm{MDI} / \mathrm{m}^{3}\right.$ bei $\left.20^{\circ} \mathrm{C}\right)$. keine Polymerisationsreaktionen in der Gasphase zu erwarten sind. Eine Bildung von MDA kann daher nicht a priori ausgeschlossen werden. Es wurde deshalb eine Analysenmethode gewählt, bei der MDI und MDA gemeinsam (als MDA) bestimmt werden. Die Methode sollte zudem MDI/MDA selektiv erfassen, um die Vortäuschung einer Isocyanat-Emission durch Amine aus anderen Quellen zu verhindern. Die Methode wurde bei Emissionsmessungen an PMDI-gebundenen Spanplatten und 
an einem Polyurethan-Klebstoff zur Herstellung tragender Holzbauteile angewendet.

3

\section{Methode}

3.1

\section{Beschreibung der Methode}

Die Methode wurde der VDI-Richtlinie 2467 (1991) zur Bestimmung von gasförmigen aliphatischen Aminen angelehnt, um sie als allgemeinere Methode auch für andere Fragestellungen und Anwendungen einsetzen zu können. Exposition der Spanplatten und Probenahme sind mit den gleichen Apparaturen und Parametern wie für die FormaldehydBestimmung z.B. gemäß DIN 55666 (Entwurf 1988) möglich.

Die zu analysierende Gasphase wird durch verdünnte Salzsäure geleitet, wobei MDA in Form des Hydrochlorids zurückgehalten wird. Gleichzeitig findet eine Hydrolyse von MDI zu MDA statt. Nach dem Eindampfen der

Absorptionslösung wird das MDA mit 2,4-Dinitro-1-fluor-ben$\mathrm{zol}(\mathrm{DNFB})$ in das Bis-2,4-dinitrophenyl-derivat (MDA-DNP $\left.{ }_{2}\right)$ überführt:

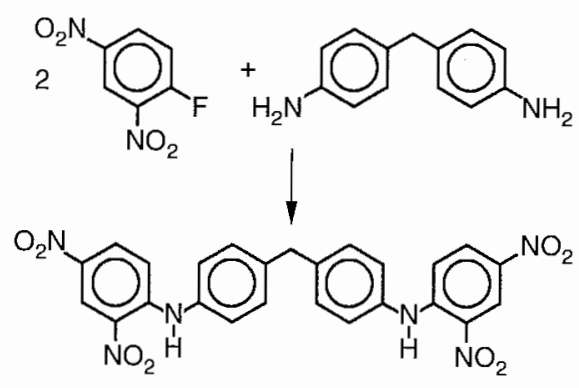

Die quantitative Bestimmung erfolgt mittels Hochleistungs Flüssigkeitschromatographie (HPLC). Die Hydrolyseprodukte von anderen verbreiteten Diisocyanaten werden ebenfalls aufgetrennt. Dadurch ist zusätzlich eine indirekte Identifizierung der emittierten Isocyanate möglich.

\section{2}

\section{Chemikalien und Geräte}

Diphenylmethan-4,4'-diisocyanat (MDI) wurde von der Bayer AG (Zürich, Schweiz) und Isophorondiamin (IPDA) von der Hüls AG (Herne, Deutschland) zur Verfügung gestellt. 4,4'Diaminodiphenylmethan (MDA), 2,4-Toluylendiamin (2,4TDA), 2,6-Toluylendiamin (2,6-TDA), Hexamethylendiamin (HDA) und 2,4-Dinitro-1-fluorbenzol (DNFB) wurden von der Fluka (Buchs, Schweiz) bezogen. Acetonitril und Wasser für die Flüssigkeitschromatographie stammten von ROMIL (Shepshed, Loughborough, Grossbritannien), resp. Merck (Darmstadt, Deutschland).

Für die Flüssigkeitschromatographie wurden Geräte der Firma Spectra Physics (San Jose, CA, USA, Modelle SP 8700 und SP 8800 ) verwendet. Die Trennsäulen wurden selbst gepackt unter Verwendung von Kartuschen $(250 \times 4 \mathrm{~mm})$ der Firma Knauer (Bad Homburg, Deutschland). Zur Methodenentwicklung wurde ein Diodenarray-UV-Detektor eingesetzt (LC-235, Perkin-Elmer, Norwalk, CT, USA). Die quantitativen Bestimmungen - vor allem im Spurenbereich - erfolgten größtenteils mit einem UV-Detektor SF 773 der Firma Kratos (ABI, Ramsey, NJ, USA). Die Proben wurden mit einem Rheodyne 7125-Injektionsventil (Cotati, CA, USA) oder bei größerem Probenanfall mit einem Autosampler (GINA 160, Gynkotek, Germering, Deutschland) aufgegeben. Die Auswertung der Chromatogramme erfolgte mit einem Trilab
2000-Datensystem (Trivector, England, Vertretung SES, Bechenheim, Deutschland). Die Spanplattenproben wurden in einer $1 \mathrm{~m}^{3}$-Klimakammer der Weiss Umwelttechnik GmbH (Reiskirchen, Deutschland) exponiert.

\section{3}

\section{HPLC}

Die Auftrennung erfolgte durch Reverse Phase-Chromatographie an LiChrosorb RP-18 (Korngröße $7 \mu \mathrm{m}$, Merck, Darmstadt, Deutschland). Als mobile Phase diente Acetonitril/Wasser im Verhältnis 75:25 mit einer Flußrate von 1 $\mathrm{ml} / \mathrm{min}$. Die Einspritzmengen betrugen $2-10 \mu \mathrm{l}$. Detektionswellenlänge war $360 \mathrm{~nm}$. Die Quantifizierung erfolgte durch externe Kalibration mit Standardlösungen von DNP-MDA in Acetonitril. Die Standardlösungen wurden durch Einwaage von Referenzderivat oder Derivatisierung bekannter Mengen MDA hergestellt.

\section{4}

\section{Herstellung der DNP-Referenzderivate}

In einem $250 \mathrm{ml}$-Rundkolben wurde das Diamin (200 - $250 \mathrm{mg}$, 1,3 - 1,7 mMol) in Acetonitril (50 ml) gelöst und mit Natriumhydrogencarbonat-Lösung ( $50 \mathrm{ml}, 1,0 \mathrm{~g} \mathrm{NaHCO}_{3}$ in $100 \mathrm{ml}$ bidest. Wasser) versetzt. Nach Zugabe von 2,4-Dinitro-1-fluorbenzol in Acetonitril (auf das Diamin bezogen doppelte Molmenge und zusätzlich $0,6-0,7 \mathrm{mMol}$ in $25 \mathrm{ml}$ ) wurde die Reaktionslösung während $1 \mathrm{~h}$ im Wasserbad bei $60^{\circ} \mathrm{C}$ gehalten. Nach Abziehen des Acetonitrils am Rotationsverdampfer unter reduziertem Druck wurde die wäßrige Phase im Eisbad gekühlt, durch eine Glasfilternutsche der Porosität 4 filtriert und der Niederschlag mit eiskaltem Wasser und wenig eiskaltem Ethanol gewaschen.

Zur Umkristallisation wurden die Niederschläge von MDA und IPDA in Toluol gelöst und die Lösung durch eine Glasfilternutsche filtriert. Die IPDA-DNP 2 -Lösung wurde anschliessend bei der Siedetemperatur des Toluols eingeengt, bis die ersten Kristalle ausfielen, während zur siedenden MDA$\mathrm{DNP}_{2}$-Lösung langsam Ethanol zugegeben wurde bis sich ein bleibender Niederschlag bildete. Die Kristalle wurden abgenutscht, mit wenig eiskaltem Ethanol gewaschen und im Vakuumexsikkator getrocknet. Die Schmelzpunkte nach zweimaligem Umkristallisieren betrugen $181-194^{\circ} \mathrm{C}$ (IPDA-DNP ${ }_{2}$ ) und $202{ }^{\circ} \mathrm{C}\left(\mathrm{MDA}^{-\mathrm{DNP}_{2}}\right)$.

Das DNP ${ }_{2}$-Derivat von HDA löste sich außer in Dimethylsulfoxid (DMSO) und in geringem Maß in Dioxan in keinem der üblichen Lösemittel. Da sich DMSO zur Umkristallisation wenig eignet, exfolgte die Reinigung des Niederschlags durch vierstündiges Extrahieren mit Dioxan unter leichtem Rückfluß (Schmelzpunkt HDA-DNP 2 : 205-206 ${ }^{\circ} \mathrm{C}$ ). 2,4-TDA und 2,6-TDA wurden unter den vorliegenden Bedingungen nur unvollständig derivatisiert (s. auch Diskussion Abschnitt 5.2).

\section{5}

\section{Probenahme}

Der Aufbau der Sammelapparatur ist aus Bild 2 ersichtlich. Das erste Absorptionsgefäß enthielt Salzsäure $(20 \mathrm{ml}, c=0,1 \mathrm{~mol} / \mathrm{l}$, $2,5 \mathrm{ml}$ Salzsäure (37\%) mit bidest. Wasser auf $250 \mathrm{ml}$ aufgefüllt). Ein zweites leeres Absorptionsgefäß dient zum Auffangen von eventuell mitgerissener Absorptionslösung.

Das Abscheideverhalten der Sammelapparatur wurde mit einem MDI-enthaltenden Luftstrom überprüft. Das MDI wurde in einem leeren Absorptionsgefäß vorgelegt und in einem Heizbad auf $140^{\circ} \mathrm{C}$ erwärmt. Über Kieselgel gereinigte und getrocknete Luft wurde zur Dotierung über das MDI geleitet und durchströmte anschließend drei hintereinander geschaltete und mit Salzsäure beschickte Absorptionsgefäße. 


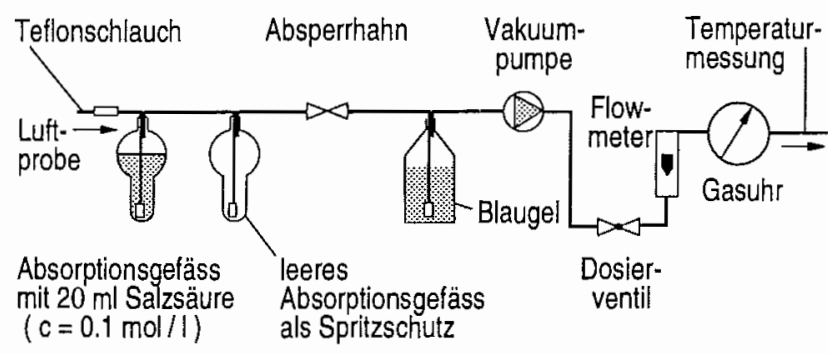

Bild 2. Sammelapparatur

Fig. 2. Sampling device

Durchflußrate (2 1/min) und Sammelzeit (25 min) entsprachen den für die Spanplattenmessungen vorgesehenen Bedingungen. Die Glasteile, die mit MDI in Berührung kamen, wurden zur Desaktivierung silanisiert, indem sie während 5 min einer 0,5\%igen Lösung (gew/vol) von N,O-Bis-trimethylsilyl-acetamid (BSA) in Dichlormethan ausgesetzt wurden.

\section{6}

\section{Analytische Derivatisierung}

Die Absorptionslösung wurde nach der Probenahme in einen 100 ml-Rundkolben überführt und das Absorptionsgefäss mit Acetonitril $(20 \mathrm{ml})$ nachgewaschen. Die vereinigten Lösungen wurden am Rotationsverdampfer bei reduziertem Druck eingeengt, unter Nachwaschen mit Acetonitril in einen $10 \mathrm{ml}$ Spitzkolben transferiert und zur Trockne eingedampft. Ein gelegentlich zurückbleibender schwer abdampfbarer Rest von Salzsäure wurde durch erneute Zugabe von Acetonitril azeotrop abdestilliert. Die vollständige Entfernung der Salzsäure ist wichtig, da die Derivatisierungsreaktion nur in basischem Milieu vollständig und reproduzierbar abläuft.

Der Abdampfrückstand wurde mit bidest. Wasser $(100 \mu 1)$ und Natrium-hydrogencarbonat-Puffer $\left(100 \mu \mathrm{l}, 1,0 \mathrm{~g} \mathrm{NaHCO}_{3}\right.$ in $100 \mathrm{ml}$ bidest. Wasser) versetzt und eine Lösung von 2,4Dinitro-1-fluor-benzol in Acetonitril zugegeben ( $40 \mu \mathrm{l}, 290-$ $300 \mathrm{mg}$ in $5 \mathrm{ml}$ Acetonitril, die Reagenslösung war bei Raumtemperatur mindestens drei Monate haltbar). Der Spitzkolben wurde mit einem Glasstopfen verschlossen und im Wasserbad $1 \mathrm{~h}$ bei $60^{\circ} \mathrm{C}$ gehalten.

Die Reaktionslösung wurde in einem Messkolben mit Acetonitril auf das gewünschte Analysenvolumen gebracht und direkt zur HPLC-Bestimmung eingesetzt. Als günstig hat sich eine Konzentration von ca. $10 \mu \mathrm{g} \mathrm{Amin} / \mathrm{ml}$ erwiesen. Proben mit unbekannter Konzentration wurden in $1 \mathrm{ml}$ gelöst. Bei manchen Probenlösungen bildete sich ein weisser Niederschlag, was sich jedoch nicht störend auf die Analyse auswirkte. Vor jeder Analysenserie wurden zur Reinheitskontrolle von Lösemitteln, Reagenzien und Hilfsmitteln 1-2 Leerproben (20 $\mathrm{ml}$ Absorptionslösung und $20 \mathrm{ml}$ Acetonitril) aufgearbeitet.

\section{4}

\section{Messungen}

\section{1}

\section{PMDI-Spanplatten}

Die Messungen erfolgten unter Verwendung einer $1 \mathrm{~m}^{3}$ Klimakammer. Die Spanplatten-Probe bestand aus vier $50 \times 50$ $\mathrm{cm}^{2}$ großen und $13 \mathrm{~mm}$ dicken Abschnitten, entsprechend einer Raumbeladung von $2 \mathrm{~m}^{2}$ pro Kubikmeter (ohne Einbezug der Schnittkanten). Die Probe wurde wie vom Hersteller angeliefert ohne Vorkonditionierung und ohne Abdeckung der Schnittkanten exponiert. Mit einer Flußrate von $21 /$ min wurden jeweils 50 Liter Kammerluft gesammelt. Das Sammelvolumen wurde bewußt tief gehalten, um ein eventuelles
Sättigungsgleichgewicht in der Klimakammer möglichst wenig zu beeinflussen.

Vor der Beschickung wurde der MDI/MDA-Untergrund in der Kammer bestimmt. Dazu wurden unmittelbar nach dem Verschliessen der Kammer und nochmals nach $24 \mathrm{~h}$ je zwei Blindproben gezogen. Die Blindproben wurden mit parallel dazu aufgearbeiteten Leerproben (Absorptions- und Waschlösung ohne Luftdurchsatz) verglichen. Zwischen Blind- und Leerproben war kein Unterschied feststellbar.

Bei den erwarteten geringen MDI/MDA-Anteilen wurde die Exponierung ohne Luftwechsel in der Kammer durchgeführt. Auch auf eine Umwälzung der Luft wurde verzichtet, da dies wegen des zusätzlichen Ventilatorteils eine ca. 50\%ige Vergrößerung des Kammervolumens bewirkt hätte. Während der einwöchigen Prüfperiode betrug die Kammertemperatur 22,0 bis $-22,3^{\circ} \mathrm{C}$. Die Luftfeuchtigkeit wurde nicht extern gesteuert und lag konstant bei $43-44 \%$ r. F. Die Probenahmen erfolgten 24, 48 und 72 Stunden sowie 7 Tage nach der Beschickung.

\section{2}

\section{Polyurethan-Klebstoff}

Bei der Probe handelte es sich um einen EinkomponentenKlebstoff für den tragenden Holzleimbau. Die Prüfung wurde unter "worst case"-Bedingungen durchgeführt, wie offene Klebflächen, dreifache Schichtdicke gegenüber der industriellen Anwendung, hohe Kammerbelegung und Beginn der Messungen sofort nach dem Klebstoffauftrag.

Als Träger für die Klebstoffschicht wurden klimatisierte Fichtenholzbrettchen $\left(20^{\circ} \mathrm{C} / 65 \%\right.$ r. F.) mit den Abmessungen $150 \times 150 \times 5 \mathrm{~mm}^{3}$ verwendet. Für den Versuch wurden 7 Brettchen mit je ca. 13,5 g Klebstoff beschichtet, was einem Auftrag von ca. $600 \mathrm{~g} / \mathrm{m}^{2}$ entspricht. Als Testzelle diente ein Glas-Exsikkator mit einem Volumen von ca. 10 Litern. Bei einer Klebfläche von $0,16 \mathrm{~m}^{2}$ ergab sich eine Kammerbelegung von $16 \mathrm{~m}^{2} / \mathrm{m}^{3}$.

Für die Probenahmen wurde Raumluft $\left(25^{\circ} \mathrm{C} / 65 \%\right.$ r. F.) mit einer Durchflussrate von $11 / \mathrm{min}$ durch die Kammer gezogen und während einer Stunde gesammelt. Vor Versuchsbeginn wurde der Kammeruntergrund (Blindwert) durch eine einstündige Exposition der unbeschichteten Fichtenholzbrettchen bestimmt. Während der ersten sechs Stunden nach der Kammerbeschickung wurden kontinuierlich Proben gezogen. Weitere Probenahmen erfolgten nach 24 und 48 Stunden. Zwischen den Probenahmen fand kein Luftaustausch statt. Als Referenz wurden $0,5 \mu \mathrm{g}$ MDA in die Absorptionslösung dosiert und wie die Prüflösungen behandelt.

\section{5}

\section{Ergebnisse und Diskussion}

5.1

\section{Ergebnisse der Messungen}

Bei den Expositionsversuchen mit Spanplatten konnte keine MDI/MDA-Emission nachgewiesen werden. Die Nachweisgrenze lag bei ca. $2 \mu \mathrm{g} \mathrm{MDA} / \mathrm{m}^{3}$ ( 0,24 vol-ppb). Sie wurde durch eine unbekannte, in den Leer- und Blindproben auftretende Komponente bestimmt, die sich chromatographisch nicht vollständig vom MDA-Derivat abtrennen ließ und deshalb als MDI/MDA-Blindwert behandelt wurde. Der Nachweisgrenze wurde der doppelte Blindwert zugrunde gelegt.

Der Polyurethan-Klebstoff gab während der ersten beiden Stunden nach der Kammerbeschickung geringe Mengen von MDI/MDA ab. Die Konzentration lag mit ca. 0,4-0,5 $\mu \mathrm{g}$ $\mathrm{MDA} / \mathrm{m}^{3}(0,05-0,06$ vol-ppb) im Bereich der Nachweisgrenze. Diese wurde wie bei den Spanplattenmessungen durch eine in 
der Blindprobe auftretende Komponente mit ähnlicher Retentionszeit wie MDA-DNP vorgegeben. In den übrigen analysierten Proben (dritte und vierte Stunde und nach 24 und 48 Stunden) war kein MDI/MDA mehr nachweisbar.

\section{2}

\section{Referenzderivate von MDA, HDA und IPDA}

In der Literatur konnten keine Angaben zu den Schmelzpunkten der DNP-Derivate gefunden werden. Die Identifizierung der Reaktionsprodukte erfolgte massenspektrometrisch, sowie IR- und UV-spektroskopisch. Die Reinheit wurde mittels HPLC überprüft.

Das DNP-Derivat von MDA fiel bei der Umkristallisation in zwei unterschiedlichen Kristallformen aus. Zuerst bildeten sich dunkelrote Plättchen und anschliessend ein orangenfarbener amorpher Niederschlag. Wurden die roten Kristalle zerrieben, entstand ein ebenfalls orangenfarbenes Pulver, das sich unter dem Mikroskop nicht vom amorphen Niederschlag unterschied. Schmelzpunkt und IR-Spektrum der beiden Kristallisationsformen stimmten ebenfalls überein, so daß das Vorliegen eines einheitlichen Derivatisierungsprodukts angenommen werden kann.

Unter dem Mikroskop waren im Kristallisat des $\mathrm{DNP}_{2}-$ Derivats von Isophorondiamin zwei deutlich voneinander abgesetzte Schmelztemperaturen (ca. $181^{\circ} \mathrm{C}$ und $188^{\circ} \mathrm{C}$ ) zu erkennen. Der Grund dürfte darin liegen, daß Isophorondiamin als ein Gemisch von cis- und trans-Isomer vorliegt. Die beiden Stereoisomere werden auch chromatographisch aufgetrennt (Bild 3).

Von 2,4-TDA und 2,6-TDA reagierte unter den vorliegenden Bedingungen nur eine der beiden Aminogruppen.

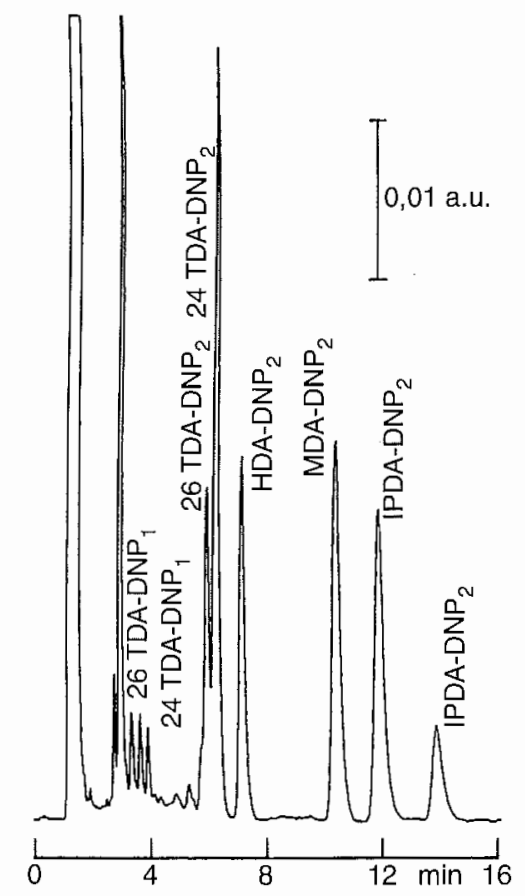

Bild 3. HPLC-Auftrennung der untersuchten Diamine (DinitrophenylDerivate aus der Derivatisierung von je ca. $50 \mu \mathrm{g}$ Diamin, Konzentration der Lösung ca. $20 \mu \mathrm{g}$ Diamin $/ \mathrm{ml}$, Einspritzmenge $4 \mu \mathrm{l}$ ). Chromatographische Bedingungen: LiChrosorb RP-18, $7 \mu \mathrm{m}, 250 \times 4$ $\mathrm{mm}$, Acetonitril/Wasser 75:25, $1 \mathrm{ml} / \mathrm{min}$, UV-Detektion bei $360 \mathrm{~nm}$ (A.U.: Absorbance Units)

Fig. 3. HPLC separation of the diamines included in the experiments (dinitrophenyl derivatives from the derivatization of $50 \mu \mathrm{g}$ of each diamine. Concentration of the sample solution ca. $20 \mu \mathrm{g}$ diamine $/ \mathrm{ml}$, injection volume $4 \mu \mathrm{l}$ ). Chromatographic conditions: LiChrosorb RP$18,7 \mu \mathrm{m}, 250 \times 4 \mathrm{~mm}$, acetonitrile/water $75: 25,1 \mathrm{ml} / \mathrm{min}$, UV-detection at $360 \mathrm{~nm}$ (A.U.: Absorbance Units)
Derivatisierungsversuche im analytischen Maßstab ergaben bei einem auf die Aminogruppen bezogenen 40fachen molaren Reagensüberschuß immer noch große Anteile an einfach phenyliertem TDA. Die Ursache dürfte bei der stark desaktivierenden Wirkung der Dinitrophenyl-Gruppe liegen. Im Gegensatz zu den anderen untersuchten Diaminen sind bei den TDAIsomeren die Aminogruppen über ein konjugiertes Doppelbindungs-System miteinander verbunden, wodurch beim einfach phenylierten TDA die Dinitrophenylgruppe mit der freien Aminogruppe in Wechselwirkung treten kann. Die Elektronendichte der Aminogruppe scheint dadurch so weit erniedrigt zu werden, daß die nukleophile Substitution des Fluors beim zweiten Derivatisierungsschritt entscheidend erschwert wird. Der Einfluß einer höheren Reaktionstemperatur und/oder längeren Reaktionszeit auf die Umsetzungsrate wurde nicht untersucht.

\section{3}

\section{HPLC}

Alle in die Untersuchung einbezogenen Diamine konnten aufgetrennt werden (Bild 3). Zusammen mit dem Amin-spezifischen Derivatisierungsschritt und der selektiven Detektionswellenlänge von $360 \mathrm{~nm}$ ergibt sich eine Bestimmungsmethode, die nicht nur zur MDI/MDA-Quantifizierung, sondern auch zur Verifizierung und Identifizierung der eingesetzten Isocyanate geeignet ist.

\section{4}

\section{Probenahme}

Bei zwei Versuchen zur Uberprüfung des Abscheideverhaltens von MDI mit der im Abschnitt 3.5 beschriebenen Apparatur wurden in den ersten Absorptionsgefäßen $12 \mu \mathrm{g}$ und $15 \mu \mathrm{g}$ MDA gefunden, während bereits in den zweiten Absorptionsgefäßen kein MDA mehr nachweisbar war. Bei einer Nachweisgrenze der Methode von $<0,05-0,1 \mu \mathrm{g}$ MDA entspricht dies einer Abscheiderate von $>99 \%$. Auf das Nachschalten eines zweiten Absorptionsgefäßes konnte deshalb bei den Probenahmen verzichtet werden.

\section{5}

\section{Analytische Derivatisierung}

Die Derivatisierung erfolgte nach der VDI-Richtlinie für aliphatische Amine, wobei die angegebenen Lösemittel- und Reagenzienmengen zur Erhöhung der Nachweisempfindlichkeit auf einen Fünftel reduziert wurden.

Die Derivatisierung wurde mit MDA-Mengen zwischen 0,5 $\mu \mathrm{g}$ und $40 \mu$ g getestet. Bei einem Teil der Versuche wurde das Amin in Salzsäure $(10 \mathrm{ml}, c=0,1 \mathrm{~mol} / \mathrm{l})$ gelöst und am Rotationsverdampfer zur Trockne eingedampft. Versuche mit dazwischen geschalteten Leerproben zeigten, daß bei der Probenaufarbeitung am Rotavap keine Verschleppung von MDA auftritt. Derivatisierungsversuche mit $4 \mu \mathrm{g}$ MDA ergaben eine mittlere Wiederfindungsrate von $69 \%$ wenn das Amin in $10 \mathrm{ml}$ Absorptionslösung dosiert wurde und von $86 \%$ bei einer direkten Derivatisierung. Bei Derivatisierungsversuchen mit 40 $\mu \mathrm{g}$ MDA wurden auch aus der Absorptionslösung 100\%ige Ausbeuten erreicht.

Zur Abschätzung der Empfindlichkeit der Methode wurden in einem Doppelversuch 0,5 $\mu \mathrm{g}$ MDA in $10 \mathrm{ml}$ Absorptionslösung gegeben und nach dem Standardverfahren derivatisiert. Gleichzeitig wurden zwei Leerproben aufgearbeitet. Ein Vergleich der Chromatogramme (Bild 4) zeigt, daß wie bei den Emissionsmessungen die Nachweisgrenze durch die unbekannte Verbindung im Chromatogramm der Leerprobe bestimmt wird. Da die Störkomponente mit den Reagenzien oder Lösemitteln eingetragen wird, könnten die in den 


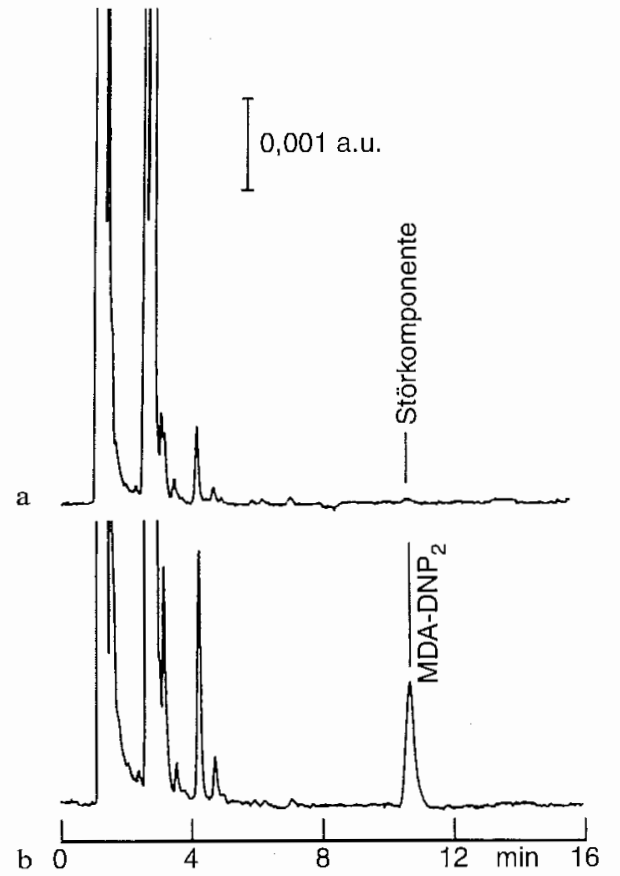

Bild 4. HPLC-Chromatogramm einer Derivatisierung mit 0,5 $\mu \mathrm{g}$ MDA. Chromatographische Bedingungen wie Bild 3. a $10 \mathrm{ml}$ Absorptionslösung mit $0,5 \mu \mathrm{g}$ MDA dotiert und nach der Vorschrift im Text aufgearbeitet, b gleich behandelte Leerprobe; die Störkomponente wurde bei den Bestimmungen als MDA angenommen und die doppelte Konzentration als Nachweisgrenze definiert

Fig. 4. HPLC-chromatogram of the derivatization of $0.5 \mu \mathrm{g} \mathrm{MDA}$; chromatographic conditions same as in Fig. 3. a $10 \mathrm{ml}$ absorption solution spiked with $0.5 \mu \mathrm{g}$ MDA and derivatized according to the procedure in the experimental section, $\mathbf{b}$ same amount of unspiked absorption solution; the marked peak was also found as background in the chamber experiments; it was treated as MDA and the detection limit defined as twice its concentration

Versuchen festgestellten Nachweisgrenzen von 2 und $0,5 \mu \mathrm{g}$ $\mathrm{MDA} / \mathrm{m}^{3}$ (0,05-2 vol-ppb) durch Reinigung der Reagenzien oder Lösemittel, Verminderung der Reagenzienmenge oder Erhöhung des Sammelvolumens herabgesetzt werden.

Daß MDA schon bei geringem Reagensüberschuß vollständig zum Bisphenyl-Derivat umgesetzt wird, zeigte eine Versuchsreihe, bei der variable Mengen MDA (50 $\mu \mathrm{g}-1000 \mu \mathrm{g}$, $0,25-5,0 \mu \mathrm{Mol})$ einer konstanten Reagensmenge $(2,4 \mathrm{mg}$ DNFB, $13 \mu \mathrm{Mol}$ ) zugesetzt und die Reaktionsausbeute bestimmt wurde. Erst bei der höchsten Diaminkonzentration

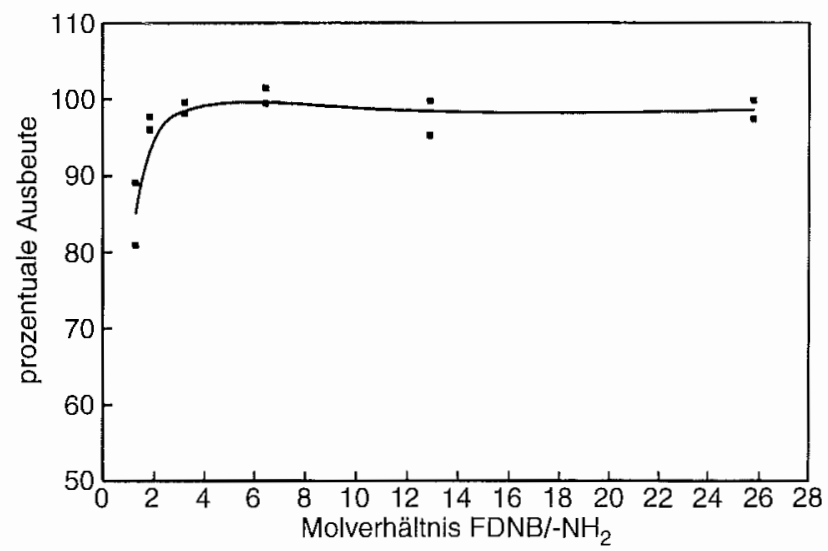

Bild 5. Einfluß des Reagenzüberschusses auf die Reaktionsausbeute (Doppelversuche). $50-1000 \mu \mathrm{g}$ MDA wurden mit einer konstanten Reagenzmenge von 2,4 mg DNFB derivatisiert

Fig. 5. Reaction yield as a function of reagent excess. $50-1000 \mu \mathrm{g}$ MDA were derivatized with a constant amount of $2.4 \mathrm{mg} 2,4$-dinitro-1fluoro-benzene mit einem Molverhältnis von Reagens zu Aminogruppen von 1,3 fiel die Reaktionsausbeute ab (Bild 5). Zugleich trat im Chromatogramm der Reaktionslösung ein zusätzlicher Peak auf, der aufgrund der Retentionszeit dem einfach phenyliertem MDA zugeordnet wurde. Schon bei der nächstkleineren Aminkonzentration (Molverhältnis 1,8) fanden sich nur noch Spuren des einfach phenylierten Derivats. Unter den StandardBedingungen (Abschnitt 3.6) können somit bis zu $650 \mu \mathrm{g}$ MDA derivatisiert werden.

HDA, IPDA, 2,4-TDA und 2,6-TDA wurden in die Derivatisierungsversuche miteinbezogen, da die ihnen zugrunde liegenden Isocyanate zusammen mit MDI am häufigsten zur Polyurethan-Herstellung eingesetzt werden. Primär war nicht an einer quantitativen Bestimmung dieser Amine gelegen, sondern an der Möglichkeit ihrer Identifizierung im Gemisch mit MDA. Obwohl nicht zur Spanplattenherstellung verwendet, sollte aufgrund ihrer weiten Verbreitung die Abtrennung dieser Amine bei einer MDI/MDA-Bestimmung gewährleistet sein. Eine Identifizierung der beiden TDA-Isomere ist trotz der Bildung von einfach und doppelt phenylierten Derivaten möglich, nicht jedoch die quantitative Bestimmung. Die

Derivatisierung von HDA und IPDA verlief dagegen quantitativ zum Bisphenylderivat. Die vorliegende Methode ist deshalb auch für die Analyse dieser beiden Diamine, resp. Diisocyanate, geeignet.

\section{Literatur}

DFG, Deutsche Forschungsgemeinschaft 1992: Gesundheitsschädliche Arbeitsstoffe, toxikologisch-arbeitsmedizinische Begründung von MAK-Werten. Verlag Chemie, Weinheim

DIN 55666 (Entwurf) 1988: Bestimmung der Ausgleichskonzentration an Formaldehyd in einem kleinen Prüfraum. Deutsches Institut für Normung eV, Berlin

Ernst, K. 1985: Erfahrungen mit Isocyanaten in der Spanplattenindustrie. Holz Roh-Werkstoff 43: 423-427

Frisch, KC.; Rumao, LP.; Pizzi, A. 1983: Diisocyanates as wood adhesives. In: Pizzi A (Hrgb.) Wood adhesives - Chemistry and Technology. Marcel Dekker, New York Basel: 289-317

Hinterwaldner, R.; Kreibich RE. 1987: Innovationschanchen im Holzleimbau. Holz-Zentralblatt 31: 421-423

Jost, M.; Rüegger, M.; Hofmann, M. 1990: Isocyanatbedingte Atemwegserkrankungen in der Schweiz. Schweiz. med. Wschr. 120: 1339-1347

Kehl, SK. 1990: Erfassung und Beurteilung der Belastung durch Isocyanate am Arbeitsplatz sowie im Wohnbereich. Diss. ETH Nr. 9222, Eidgenössische Technische Hochschule, Zürich

Marutzky, R.; Menzel, W. 1985: Bestimmung der nachträglichen Abgabe von Diphenylmethandiisocyanat (MDI) und Diaminodiphenylmethan (MDA) aus mit PMDI gebundenen Spanplatten. WKI-Kurzbericht Nr. 28/85, Fraunhofer-Institut für Holzforschung, Braunschweig

Radovic, B.; Goth, H. 1994: Einkomponenten-Polyurethan-Klebstoffe für die Herstellung von tragenden Holzbauteilen. Bauen mit Holz 1: 22-32

Schmidtke, F.; Seifert, B. 1990: A highly sensitive high-performance liquid chromatographic procedure for the determination of isocyanates in air. Fresenius J. Anal. Chem 336: 647-654

Schriever, E. 1986: Diisocyanat- und Polyurethanklebstoffe für Holz und Holzwerkstoffe. WKI-Bericht Nr. 14, 2. Auflage, FraunhoferInstitut für Holzforschung, Braunschweig

Schwarz, J. 1991: Oekologie im Bau. Verlag Paul Haupt, Bern Stuttgart VDI-Richtlinie 2467/Blatt 2 1991: Messen der Konzentration primärer und sekundärer aliphatischer Amine mit der HochleistungsFlüssigkeits-Chromatographie (HPLC), Kommission Reinhaltung der Luft im VDI und DIN, Verein Deutscher Ingenieure, Düsseldorf 